Hardy-Ramanujan Journal

Vol.24 (2001) 2-8

\title{
ON SOME RAMANUJAN'S P-Q IDENTITIES
}

\author{
H. S. Madhusudhan, M. S. Mahadeva Naika and K. R. Vasuki*
}

\begin{abstract}
In this paper we obtain some P-Q eta-function identities of Ramanujan on employing some modular equations in Ramanujan's alternative theory of elliptic functions of signature 4 .
\end{abstract}

Key words: Elliptic functions, Modular equations

2000 AMS Mathematics Subject Classification: 33D15, 33D20

\section{Introduction}

Srinivasa Ramanujan has recorded several results in the theories of elliptic functions to alternative bases corresponding to the classical theory in his Second Notebook [6, pp. 257$262]$.

K. Venkatachaliengar [8, pp. 89-95] examined some of the Entries in Ramanujan's Notebooks [6] devoted to alternative theories. In order to establish several beautiful formulas for $1 / \pi$ stated by Ramanujan [5], [7, pp.23-29], J. M. Borwein and P. B. Borwein [4, pp. 177-185] have developed the "corresponding theories".

In Entries 51-72 of Chapter 25 of the Second Notebook [6], Ramanujan states twentythree elegant, so-called, P-Q identities. Eighteen of these P-Q identities have been proved by B. C. Berndt [1, pp. 204-237] and B. C. Berndt and L.-C. Zhang [3] on employing various modular equations belonging to the theory of signature 2 (classical theory). The main purpose of this paper is to establish some P-Q identities stated by Ramanujan on employing various modular equations belonging to the theory of signature 4 .

\section{Some Preliminary Results}

First we shall provide some useful notations and definitions. As customary, for any complex number $a$ set

$$
(a ; q)_{\infty}=\prod_{k=0}^{\infty}\left(1-a q^{k}\right),|q|<1
$$


Following Ramanujan, we define

$$
f\left(-q=(q ; q)_{\infty}\right.
$$

Let

$$
z(r):=z(r ; x):={ }_{2} F_{1}\left(\frac{1}{r}, \frac{r-1}{r} ; 1 ; x\right)
$$

and

$$
q_{r}:=q_{r}(x):=\exp \left[-\pi \csc \left(\frac{\pi}{r}\right) \frac{{ }_{2} F_{1}\left(\frac{1}{r}, \frac{r-1}{r} ; 1 ; 1-x\right)}{{ }_{2} F_{1}\left(\frac{1}{r}, \frac{r-1}{r} ; 1 ; x\right)}\right],
$$

where $r=2,3,4,6$ and $0<x<1$,

$$
{ }_{2} F_{1}(a, b ; c ; x)=\sum_{k=0}^{\infty} \frac{(a)_{k}(b)_{k}}{(c)_{k} k !} x^{k}
$$

with $(a)_{k}=(a)(a+1) \ldots(a+k-1)$.

Let $n$ denote a fixed natural number and assume that

$$
n \frac{{ }_{2} F_{1}\left(\frac{1}{r}, \frac{r-1}{r} ; 1 ; 1-\alpha\right)}{{ }_{2} F_{1}\left(\frac{1}{r}, \frac{r-1}{r} ; 1 ; \alpha\right)}=\frac{{ }_{2} F_{1}\left(\frac{1}{r}, \frac{r-1}{r} ; 1 ; 1-\beta\right)}{{ }_{2} F_{1}\left(\frac{1}{r}, \frac{r-1}{r} ; 1 ; \beta\right)}
$$

where $r=2,3,4$ or 6 . Then a modular equation of degree $n$ in the theory of elliptic functions of signature $r$ is a relation between $\alpha$ and $\beta$ induced by (2.1). We often say that $\beta$ is of degree $n$ over $\alpha$ and $m(r):=\frac{z(r ; \alpha)}{z(r ; \beta)}$ is called the multiplier.

We also use the notations $z_{1}:=z_{1}(\alpha):=z(r ; \alpha)$ and $z_{n}:=z_{n}(\beta):=z(r ; \beta)$ to indicate that $\beta$ has degree $n$ over $\alpha$. When the context is clear, we omit the argument $r$ in $q_{r}, z(r)$ and $m(r)$.

We now collect in the following theorem some of Ramanujan's modular equations belonging to the theory of signature 4 .

Theorem 2.1. The following modular equations hold in the theory of signature 4.

(i) if $\beta$ is of degree 3 over $\alpha$ then,

$$
m^{2}=\left(\frac{\beta}{\alpha}\right)^{1 / 2}+\left(\frac{1-\beta}{1-\alpha}\right)^{1 / 2}-\frac{9}{m^{2}}\left(\frac{\beta(1-\beta)}{\alpha(1-\alpha)}\right)^{1 / 2} .
$$

(ii) If $\beta$ is of degree 5 over $\alpha$, then

$$
m=\left(\frac{\beta}{\alpha}\right)^{1 / 4}+\left(\frac{1-\beta}{1-\alpha}\right)^{1 / 4}-\frac{5}{m}\left(\frac{\beta(1-\beta)}{\alpha(1-\alpha)}\right)^{1 / 4} .
$$


(iii) If $\beta$ is of degree 7 over $\alpha$, then

$$
\begin{aligned}
m^{2} & =\left(\frac{\beta}{\alpha}\right)^{1 / 2}+\left(\frac{1-\beta}{1-\alpha}\right)^{1 / 2}-\frac{49}{m^{2}}\left(\frac{\beta(1-\beta)}{\alpha(1-\alpha)}\right)^{1 / 2} \\
& -8\left(\frac{\beta(1-\beta)}{\alpha(1-\alpha)}\right)^{1 / 6}\left[\left(\frac{\beta}{\alpha}\right)^{1 / 6}+\left(\frac{1-\beta}{1-\alpha}\right)^{1 / 6}\right] .
\end{aligned}
$$

(iv) If $\beta$ is of degree 9 over $\alpha$, then

$$
\sqrt{m}=\left(\frac{\beta}{\alpha}\right)^{1 / 8}+\left(\frac{1-\beta}{1-\alpha}\right)^{1 / 8}-\frac{3}{\sqrt{m}}\left(\frac{\beta(1-\beta)}{\alpha(1-\alpha)}\right)^{1 / 8}
$$

(v) If $\beta$ is of degree 13 over $\alpha$, then

$$
\begin{aligned}
& m=\left(\frac{\beta}{\alpha}\right)^{1 / 4}+\left(\frac{1-\beta}{1-\alpha}\right)^{1 / 4}-\frac{13}{m}\left(\frac{\beta(1-\beta)}{\alpha(1-\alpha)}\right)^{1 / 4} \\
& -4\left(\frac{\beta(1-\beta)}{\alpha(1-\alpha)}\right)^{1 / 12}\left[\left(\frac{\beta}{\alpha}\right)^{1 / 12}+\left(\frac{1-\beta}{1-\alpha}\right)^{1 / 12}\right] .
\end{aligned}
$$

(vi) If $\beta$ is of degree 25 over $\alpha$, then

$$
\begin{gathered}
\sqrt{m}=\left(\frac{\beta}{\alpha}\right)^{1 / 8}+\left(\frac{1-\beta}{1-\alpha}\right)^{1 / 8}-\frac{5}{\sqrt{m}}\left(\frac{\beta(1-\beta)}{\alpha(1-\alpha)}\right)^{1 / 8} \\
-2\left(\frac{\beta(1-\beta)}{\alpha(1-\alpha)}\right)^{1 / 24}\left[\left(\frac{\beta}{\alpha}\right)^{1 / 24}+\left(\frac{1-\beta}{1-\alpha}\right)^{1 / 24}\right] .
\end{gathered}
$$

Proof. For a proof of (i) see [2, p. 156. Theorem 10.5]. For a proof of (ii) see [2, p.157, Theorem 10.6]. For proofs of (iii) and (iv) see [2, p.158, Theorm 10.7 and Theorem 10.8]. For a proof of (v) see [2, p.159, Theorem 10.9]. For a proof of (vi) see [2, p.160, Theorem 10.10].

\section{Main Theorem}

In this Section we obtain six P-Q identities (3.1)-(3.6) due to Ramanujan which appears as Entries 51,53,55,56,57 and 58 of Chapter 25 of his Second Notebook on employing Ramanujan's modular equations in the theory of signature 4.

\section{Theorem 3.1}


(i) If

$$
P=\frac{f^{2}(-q)}{q^{1 / 6} f^{2}\left(-q^{3}\right)} \text { and } Q=\frac{f^{2}\left(-q^{2}\right)}{q^{1 / 3} f^{2}\left(-q^{6}\right)},
$$

then

$$
P Q+\frac{9}{P Q}=\left(\frac{Q}{P}\right)^{3}+\left(\frac{P}{Q}\right)^{3}
$$

(ii) If

$$
P=\frac{f(-q)}{q^{1 / 6} f\left(-q^{5}\right)} \text { and } Q=\frac{f\left(-q^{2}\right)}{q^{1 / 3} f\left(-q^{10}\right)},
$$

then

$$
P Q+\frac{5}{P Q}=\left(\frac{Q}{P}\right)^{3}+\left(\frac{P}{Q}\right)^{3}
$$

(iii) If

$$
P=\frac{f^{2}(-q)}{q^{1 / 2} f^{2}\left(-q^{7}\right)} \text { and } Q=\frac{f^{2}\left(-q^{2}\right)}{q f^{2}\left(-q^{14}\right)}
$$

then

$$
P Q+\frac{49}{P Q}=\left(\frac{Q}{P}\right)^{3}+\left(\frac{P}{Q}\right)^{3}-8\left(\frac{Q}{P}\right)-8\left(\frac{P}{Q}\right)
$$

(iv) If

$$
P=\frac{f(-q)}{q^{1 / 3} f\left(-q^{9}\right)} \text { and } Q=\frac{f\left(-q^{2}\right)}{q^{2 / 3} f\left(-q^{18}\right)},
$$

then

$$
P^{3}+Q^{3}=P^{2} Q^{2}+3 P Q
$$

(v) If

$$
P=\frac{f(-q)}{q^{1 / 2} f\left(-q^{13}\right)} \text { and } Q=\frac{f\left(-q^{2}\right)}{q f\left(-q^{26}\right)},
$$

then

$$
P Q+\frac{13}{P Q}=\left(\frac{Q}{P}\right)^{3}+\left(\frac{P}{Q}\right)^{3}-4\left(\frac{Q}{P}\right)-4\left(\frac{P}{Q}\right)
$$

(vi) If

$$
P=\frac{f\left(-q^{1 / 5}\right)}{q^{1 / 5} f\left(-q^{5}\right)} \text { and } Q=\frac{f\left(-q^{2 / 5}\right)}{q^{2 / 5} f\left(-q^{10}\right)}
$$

then

$$
P^{2} Q^{2}+5 P Q=P^{3}-2 P^{2} Q-2 P Q^{2}+Q^{3} .
$$


Proof of (3.1). When $y=\pi \sqrt{2} \frac{{ }_{2} F_{1}\left(\frac{1}{4}, \frac{3}{4} ; 1 ; 1-x\right)}{{ }_{2} F_{1}\left(\frac{1}{4}, \frac{3}{4} ; 1 ; x\right)}$ and $q=e^{-y}$, we find that $[2, p .148]$

$$
q^{1 / 24} f(-q)=\sqrt{z} 2^{-1 / 4} x^{1 / 24}(1-x)^{1 / 12}
$$

and

$$
q^{1 / 12} f\left(-q^{2}\right)=\sqrt{z} 2^{-1 / 2} x^{1 / 12}(1-x)^{1 / 24} .
$$

Using the above two identities, we deduce that

$$
\sqrt{z} x^{1 / 8}=2^{3 / 4} q^{1 / 8} \frac{f^{2}\left(-q^{2}\right)}{f(-q)}
$$

and

$$
\sqrt{z}(1-x)^{1 / 8}=\frac{f^{2}(-q)}{f\left(-q^{2}\right)},
$$

If $\beta$ is of degree 3 over $\alpha$, then from (3.7) and (3.8), it follows that

$$
\begin{gathered}
\alpha^{1 / 8}=\frac{2^{3 / 4} q^{1 / 8}}{\sqrt{z}_{1}} \frac{f^{2}\left(-q^{2}\right)}{f(-q)}, \beta^{1 / 8}=\frac{2^{3 / 4} q^{3 / 8}}{\sqrt{z_{3}}} \frac{f^{2}\left(-q^{6}\right)}{f\left(-q^{3}\right)}, \\
(1-\alpha)^{1 / 8}=\frac{1}{\sqrt{z_{1}}} \frac{f^{2}(-q)}{f\left(-q^{2}\right)},(1-\beta)^{1 / 8}=\frac{1}{\sqrt{z_{3}}} \frac{f^{2}\left(-q^{3}\right)}{f\left(-q^{6}\right)},
\end{gathered}
$$

Employing (3.9) in (2.2), we obtain

$$
1=q \frac{f^{8}\left(-q^{6}\right) f^{4}(-q)}{f^{4}\left(-q^{3}\right) f^{8}\left(-q^{2}\right)}+\frac{f^{8}(-q) f^{4}\left(-q^{3}\right)}{f^{4}\left(-q^{2}\right) f^{8}\left(-q^{6}\right)}-9 q \frac{f^{4}\left(-q^{6}\right) f^{4}\left(-q^{3}\right)}{f^{4}(-q) f^{4}\left(-q^{2}\right)}
$$

Upon simplification, we find that (3.10) is same as (3.1).

Proof of (3.2). If $\beta$ is of degree 5 over $\alpha$, then from (3.7) and (3.8), it follows that

$$
\begin{gathered}
\alpha^{1 / 8}=\frac{2^{3 / 4} q^{1 / 8}}{\sqrt{z}_{1}} \frac{f^{2}\left(-q^{2}\right)}{f(-q)}, \beta^{1 / 8}=\frac{2^{3 / 4} q^{5 / 8}}{\sqrt{z_{5}}} \frac{f^{2}\left(-q^{10}\right)}{f\left(-q^{5}\right)}, \\
(1-\alpha)^{1 / 8}=\frac{1}{\sqrt{z_{1}}} \frac{f^{2}(-q)}{f\left(-q^{2}\right)},(1-\beta)^{1 / 8}=\frac{1}{\sqrt{z_{5}}} \frac{f^{2}\left(-q^{5}\right)}{f\left(-q^{10}\right)},
\end{gathered}
$$

Employing (3.11) in (2.3), we obtain

$$
1=q \frac{f^{4}\left(-q^{10}\right) f^{2}(-q)}{f^{2}\left(-q^{5}\right) f^{4}\left(-q^{2}\right)}+\frac{f^{4}\left(-q^{5}\right) f^{2}\left(-q^{2}\right)}{f^{2}\left(-q^{10}\right) f^{4}(-q)}-\frac{5}{q} \frac{f^{2}\left(-q^{10}\right) f^{2}\left(-q^{5}\right)}{f^{2}(-q) f^{2}\left(-q^{2}\right)}
$$

On simplification we see that (3.12) is same as (3.2).

Proof of (3.3). If $\beta$ is of degree 7 over $\alpha$, then from (3.7) and (3.8), it follows that

$$
\alpha^{1 / 8}=\frac{2^{3 / 4} q^{1 / 8}}{\sqrt{z}_{1}} \frac{f^{2}\left(-q^{2}\right)}{f(-q)}, \beta^{1 / 8}=\frac{2^{3 / 4} q^{7 / 8}}{\sqrt{z}_{7}} \frac{f^{2}\left(-q^{14}\right)}{f\left(-q^{7}\right)}
$$




$$
(1-\alpha)^{1 / 8}=\frac{1}{\sqrt{z_{1}}} \frac{f^{2}(-q)}{f\left(-q^{2}\right)},(1-\beta)^{1 / 8}=\frac{1}{\sqrt{z_{7}}} \frac{f^{2}\left(-q^{7}\right)}{f\left(-q^{14}\right)},
$$

Employing (3.13) in (2.4), we obtain

$$
\begin{gathered}
1=q^{3} \frac{f^{8}\left(-q^{14}\right) f^{4}(-q)}{f^{4}\left(-q^{7}\right) f^{8}\left(-q^{2}\right)}+\frac{f^{8}\left(-q^{7}\right) f^{4}\left(-q^{2}\right)}{f^{4}\left(-q^{14}\right) f^{8}(-q)}-49 q^{3} \frac{f^{4}\left(-q^{14}\right) f^{4}\left(-q^{7}\right)}{f^{4}(-q) f^{4}\left(-q^{2}\right)} . \\
-8 q \frac{f^{4 / 3}\left(-q^{14}\right) f^{4 / 3}\left(-q^{7}\right)}{f^{4 / 3}(-q) f^{4 / 3}\left(-q^{2}\right)}\left[q \frac{f^{8 / 3}\left(-q^{14}\right) f^{4 / 3}(-q)}{f^{4 / 3}\left(-q^{7}\right) f^{8 / 3}\left(-q^{2}\right)}+\frac{f^{8 / 3}\left(-q^{7}\right) f^{4 / 3}\left(-q^{2}\right)}{f^{4 / 3}\left(-q^{14}\right) f^{8 / 3}(-q)}\right] .
\end{gathered}
$$

On simplification we see that (3.14) is same as (3.3).

Proof of (3.4). If $\beta$ is of degree 9 over $\alpha$, then from (3.7) and (3.8), it follows that

$$
\begin{gathered}
\alpha^{1 / 8}=\frac{2^{3 / 4} q^{1 / 8}}{\sqrt{z}_{1}} \frac{f^{2}\left(-q^{2}\right)}{f(-q)}, \beta^{1 / 8}=\frac{2^{3 / 4} q^{9 / 8}}{\sqrt{z_{9}}} \frac{f^{2}\left(-q^{18}\right)}{f\left(-q^{9}\right)}, \\
(1-\alpha)^{1 / 8}=\frac{1}{\sqrt{z_{1}}} \frac{f^{2}(-q)}{f\left(-q^{2}\right)},(1-\beta)^{1 / 8}=\frac{1}{\sqrt{z_{9}}} \frac{f^{2}\left(-q^{9}\right)}{f\left(-q^{18}\right)},
\end{gathered}
$$

Employing (3.15) in (2.5), we obtain

$$
1=q \frac{f^{2}\left(-q^{18}\right) f(-q)}{f\left(-q^{9}\right) f^{2}\left(-q^{2}\right)}+\frac{f^{2}\left(-q^{9}\right) f\left(-q^{2}\right)}{f\left(-q^{18}\right) f^{2}(-q)}-3 q \frac{f\left(-q^{9}\right) f\left(-q^{18}\right)}{f(-q) f\left(-q^{2}\right)}
$$

Further simplification of (3.16) yields (3.4).

Proof of (3.5). If $\beta$ is of degree 13 over $\alpha$, then from (3.7) and (3.8), it follows that

$$
\begin{gathered}
\alpha^{1 / 8}=\frac{2^{3 / 4} q^{1 / 8}}{\sqrt{z_{1}}} \frac{f^{2}\left(-q^{2}\right)}{f(-q)}, \beta^{1 / 8}=\frac{2^{3 / 4} q^{13 / 8}}{\sqrt{z_{13}}} \frac{f^{2}\left(-q^{26}\right)}{f\left(-q^{13}\right)} \\
(1-\alpha)^{1 / 8}=\frac{1}{\sqrt{z_{1}}} \frac{f^{2}(-q)}{f\left(-q^{2}\right)},(1-\beta)^{1 / 8}=\frac{1}{\sqrt{z_{13}}} \frac{f^{2}\left(-q^{13}\right)}{f\left(-q^{26}\right)},
\end{gathered}
$$

Using (3.17) in (2.6), it follows that

$$
\begin{gathered}
1=q^{3} \frac{f^{4}\left(-q^{26}\right) f^{2}(-q)}{f^{2}\left(-q^{13}\right) f^{4}\left(-q^{2}\right)}+\frac{f^{4}\left(-q^{13}\right) f^{2}\left(-q^{2}\right)}{f^{2}\left(-q^{26}\right) f^{4}(-q)}-13 q^{3} \frac{f^{2}\left(-q^{26}\right) f^{2}\left(-q^{13}\right)}{f^{2}\left(-q^{2}\right) f^{2}(-q)} \\
-4 q \frac{f^{2 / 3}\left(-q^{26}\right) f^{2 / 3}\left(-q^{13}\right)}{f^{2 / 3}(-q) f^{2 / 3}\left(-q^{2}\right)}\left[q \frac{f^{4 / 3}\left(-q^{26}\right) f^{2 / 3}(-q)}{f^{2 / 3}\left(-q^{13}\right) f^{4 / 3}\left(-q^{2}\right)}+\frac{f^{4 / 3}\left(-q^{13}\right) f^{2 / 3}\left(-q^{2}\right)}{f^{2 / 3}\left(-q^{26}\right) f^{4 / 3}(-q)}\right] .
\end{gathered}
$$

Further simplification of (3.18) yields (3.5).

Proof of (vi). If $\beta$ is of degree 25 over $\alpha$, then from (3.7) and (3.8), it follows that

$$
\alpha^{1 / 8}=\frac{2^{3 / 4} q^{1 / 8}}{\sqrt{z}_{1}} \frac{f^{2}\left(-q^{2}\right)}{f(-q)}, \beta^{1 / 8}=\frac{2^{3 / 4} q^{25 / 8}}{\sqrt{z}_{25}} \frac{f^{2}\left(-q^{50}\right)}{f\left(-q^{25}\right)},
$$




$$
(1-\alpha)^{1 / 8}=\frac{1}{\sqrt{z_{1}}} \frac{f^{2}(-q)}{f\left(-q^{2}\right)},(1-\beta)^{1 / 8}=\frac{1}{\sqrt{z_{25}}} \frac{f^{2}\left(-q^{25}\right)}{f\left(-q^{50}\right)},
$$

Using (3.19) in (2.7), it follows that

$$
\begin{gathered}
1=q^{3} \frac{f^{2}\left(-q^{50}\right) f(-q)}{f\left(-q^{25}\right) f^{2}\left(-q^{2}\right)}+\frac{f^{2}\left(-q^{25}\right) f\left(-q^{2}\right)}{f\left(-q^{50}\right) f^{2}(-q)}-5 q^{3} \frac{f\left(-q^{50}\right) f\left(-q^{25}\right)}{f(-q) f\left(-q^{2}\right)} \\
-2 q \frac{f^{1 / 3}\left(-q^{50}\right) f^{1 / 3}\left(-q^{25}\right)}{f^{1 / 3}(-q) f^{1 / 3}\left(-q^{2}\right)}\left[q \frac{f^{2 / 3}\left(-q^{50}\right) f^{1 / 3}(-q)}{f^{1 / 3}\left(-q^{25}\right) f^{2 / 3}\left(-q^{2}\right)}+\frac{f^{2 / 3}\left(-q^{25}\right) f^{1 / 3}\left(-q^{2}\right)}{f^{1 / 3}\left(-q^{50}\right) f^{2 / 3}(-q)}\right] .
\end{gathered}
$$

Upon simplification we see that (3.20) is same as (3.6).

\section{Acknowledgement}

We wish to thank Dr.C.Adiga for his valuable suggestions during the preparation of this paper. The first author would like to thank Acharya Institute of Technology, Bangalore, India, for their financial support. 


\section{References}

1. B.C.Berndt, Ramanujan's Note books, Part IV, Springer-Verlag, New York 1994.

2. B.C. Berndt, Ramanujan's Notebooks, Part V, Springer-Verlag, New York, 1998.

3. B.C. Berndt and L.-C. Zhang, Ramanujan's identities for eta-functions, Math. Ann., 292 (1992), 561-573.

4. J.M.Borwein and P.B.Borwein, A cubic counterpart of Jacobi's identity and the AGM, Trans. Amer. Math.Soc., 323 (1991), 691-701.

5. S.Ramanujan, Modular equations and approximation to $\pi$, Quart.J.Math.(Oxford), 45(1914), 350-372.

6.S. Ramanujan, Notebooks (2 volumes), Tata Institute of Fundamental Research, Bombay, 1957.

7. S.Ramanujan, Collected papers,, Chelsea, New York, 1962.

8. K.Venkatchaliengar, Development of Elliptic Functions According to Ramanujan, Technical Report 2, Madurai Kamaraj University, Madurai, 1988.

* Department of Mathematics

Acharya Institute of Technology

Soldevanahalli, Chikkabanavara Post

Hesaragatta Road

Bangalore-560090

INDIA.

Department of Studies in Mathematics

University of Mysore

Manasagangotri

Mysore - 570006

INDIA. 